\section{Breastfeeding and association with refractive error in young Singapore Chinese children}

WK Sham 1, M Dirani ${ }^{2,3}$, YS Chong ${ }^{4}$,

DM Hornbeak ${ }^{5,6}$, G Gazzard ${ }^{7}$, J Li ${ }^{1}$ and SM Saw ${ }^{8}$

\begin{abstract}
Purpose The objective of this study was to investigate an association between spherical refractive error and breastfeeding.

Methods Strabismus, amblyopia, and refractive errors in Singaporean preschoolers (STARS) is a cross-sectional population-based study of 3009 Chinese children aged 6-72 months conducted between June 2006 and September 2008 in Singapore. Parents were asked about the history of breastfeeding in face-to-face interviews. Children without cycloplegia or without refraction assessment were excluded. The final sample analysed was 2639 children (1375 male, 1264 female). Results Out of those who were breastfed, $842(41.3 \%)$ were breastfed for more than 3 months and $599(29.4 \%)$ were breastfed longer than 6 months. The prevalence of myopia, defined as the spherical equivalent refraction of the right eye of at least -0.5 dioptres (D), was $11.3 \%$ (95\% CI $(10.1,12.5))$. The mean spherical equivalent refraction of breastfed children was $0.12 \mathrm{D}$ higher than that of children who were not breastfed $(P$-value $=0.03)$.

Breastfeeding, however, was not associated with myopia (adjusted OR $=0.85 ; 95 \%$ CI $(0.62,1.18)$ ). Conclusions The results show that breastfeeding is associated with more hyperopic spherical equivalent refraction in young Chinese children in Singapore. Eye (2010) 24, 875-880; doi:10.1038/eye.2009.198; published online 11 September 2009
\end{abstract}

Keywords: breastfeeding; refractive error; Singapore

\section{Introduction}

Myopia is one of the leading causes of blindness throughout the world. The myopic population is generally high worldwide. Its prevalence varies by country, ethnicity, age group, and development of the city within the country. At present, the explanatory factors for myopia remain uncertain, but many studies have suggested that genes, as well as the gene-environment interaction, will be the primary elements of refractive errors. ${ }^{1-5}$

In addition, it has been noted that nutrition intake, particularly breastfeeding, affects retinal development and eye growth, which may have a vital role in juvenile-onset myopia. ${ }^{6}$ Some studies have shown an association between breastfeeding and eye diseases such as retinopathy of prematurity, retinal detachment, and stereoacuity, whereas others have suggested otherwise. ${ }^{6-10}$ For the relationship between breastfeeding and myopia, Chong et al conducted a study of 797 Singapore children for ages 10, 11, and 12 years and showed significantly lesser myopia in children who were breastfed (adjusted OR $=0.58 ; 95 \%$

CI $(0.39,0.84))$, whereas Rudnicka et al has found a statistically insignificant result (pooled odds ratio $(\mathrm{OR})$ for reduced vision $=1.03,95 \% \mathrm{CI}$ $(0.9,1.18), P$-value for heterogeneity $=0.39$ $\left(\chi^{2}\right.$-test) for ages $10-11$ years and pooled OR for reduced vision $=1.02,95 \% \mathrm{CI}(0.89,1.16)$, $P$-value for heterogeneity $=0.88\left(\chi^{2}\right.$-test $)$ for ages 15-16 years) for the effect of breastfeeding on poor vision in British children. ${ }^{11,12}$ We aim to evaluate the association of breastfeeding with refractive errors in young Singapore Chinese children.

\section{Materials and methods}

\section{Study population}

The strabismus, amblyopia, and refractive errors in Singaporean preschoolers (STARS) study is a population-based study of Chinese children aged 6-72 months. A list of Chinese
${ }^{1}$ Faculty of Science, Department of Statistics and Applied Probability, National University of Singapore, Singapore, Singapore

${ }^{2}$ Singapore Eye Research Institute, Singapore, Republic of Singapore

${ }^{3}$ Centre for Eye Research Australia, University of Melbourne, Royal Victorian Eye and Ear Hospital, Melbourne, Victoria, Australia

${ }^{4}$ Department of Obstetrics and Gynaecology, Yong Loo Lin School of Medicine, National University of Singapore,

Singapore, Singapore

${ }^{5}$ Duke-NUS Graduate Medical School, Singapore

${ }^{6}$ Department of Ophthalmology, Duke University Medical Center, Durham, NC, USA

7 Moorfields Eye Hospital, London, UK

${ }^{8}$ Department of Community, Occupational and Family Medicine, Yong Loo Lin School of Medicine, National University of Singapore, Singapore

Correspondence: SM Saw, Department of Community, Occupational and Family Medicine, 10 Medical Drive, Yong Loo Lin School of Medicine, National University of Singapore, Singapore 117597.

Tel: + 6565168745 ;

Fax: + 6567785743

E-mail: cofsawsm@

nus.edu.sg

Received: 22 February 2009 Accepted in revised form: 2 June 2009

Published online: 11

September 2009 
households residing in the Housing Development Board (HDB) estates in the southern and western parts of Singapore was obtained from the Ministry of Home Affairs and we then recruited a total of 5648 Chinese children using disproportionate stratified (by age group) random sampling. A household that had no eligible child (aged 6-72 months), had moved away from the residing address, or was non-Chinese was considered 'ineligible' (1484 children). Out of 4164 eligible candidates, the response rate was $72.3 \%(n=3009)$. Children without cycloplegia using eye drops or those without the spherical equivalent recorded were excluded. The final sample in the analysis was 2639 children (1375 male, 1264 female).

\section{Interviews}

The childrens' parents were interviewed face to face during the STARS study on whether the child was ever breastfed or fed breast milk, the duration of breastfeeding, and to describe the type of breastfeeding as exclusive, mostly (nonformula supplements), or partly (formula supplements). In addition, the age at which the child was first fed breast milk and how the mother fed the child breast milk (direct, bottled, mixed ways) were also recorded in the questionnaire. Questions on family history, pregnancy history, history of health, and ocular condition of the child, and on outdoor, indoor, and pre-school activities were also asked during the interview.

\section{Eye examinations}

Cycloplegic refraction was carried out after $30 \mathrm{~min}$ of 3 drops of $1 \%$ cyclopentolate and $2.5 \%$ phenylephrine, with a 5 min interval. Refractive error was measured using the autokeratorefractometer (Canon Autorefractor RK-F1, Tokyo, Japan) for children aged $>24$ to 72 months, and streak retinoscopy (Welch Allyn, Paris, France) or retinomax handheld autorefraction (K-PLUS 2, Tokyo, Japan) for those aged $<24$ months. Before the study, a participant information sheet was given and explained by an Optometrist and a written consent was obtained from all children's parents. Approval was also obtained by the Ethics Committees of the Singapore Eye Research Institute and the National Healthcare Group.

\section{Statistical analyses and definitions}

The definition of myopia was spherical equivalent refraction of the right eye of at least -0.5 dioptres (D).
The correlation between the spherical equivalent refraction of the left eye and right eye within this study is 0.94 and thus only right eye data were analysed. Statistical analyses were conducted using the SAS 9.1.3 Service Pack 3 (SAS Institute Inc., Cary, NC, USA) and SPSS version 16 (SPSS Inc.). The association between the prevalence of myopia and breastfeeding or other sociodemographic variables was determined using $\chi^{2}$-test for dichotomous or any categorical variables and $t$-test for quantitative variables. For the spherical equivalent refraction of the right eye, Pearson's product-moment correlation coefficients were computed for quantitative variables. ANOVA F-test was used in replacement of $t$-test for any categorical variables with more than two groups. Subsequently, we modelled the prevalence of myopia using a multiple logistic regression model, with adjustment of gender, age (months), breastfeeding status, birth weight $(\mathrm{g})$, child's height $(\mathrm{cm})$, time spent outdoors (per day), maternal age (years), mother's educational status, mother's smoking history, and parental myopia status. In addition, we modelled the spherical equivalent refraction of the right eye using a multiple linear regression model with adjustment of the same factors. Statistical significance was defined as $P$-value $<0.05$.

\section{Results}

There are 3009 participants (1570 males, 1439 females) and 1155 non-participants (227 males, 199 females, 729 missing values). No statistical differences were observed between the participants and non-participants when we compared them by gender, age, and age group. However, the districts the participants resided in were significantly different from those of the non-participants $(P$-value $<0.001)$.

Out of those enrolled in the study, there were 2639 dilated cases with measurements of spherical equivalent refraction recorded (1375 males, 1264 females). Prevalence of myopia was $11.3 \%$ (299 of $2639 ; 95 \%$ CI $(10.1,12.5))$. A large percentage $(2040(77.3 \%))$ of the 2639 dilated children were breastfed, with $842(41.3 \%)$ breastfed for more than 3 months and 599 (29.4\%) breastfed longer than 6 months. In addition, 424 children were breastfed exclusively. The age range of participants was between 6 and 72 months when tested. A graph showing the distribution of refractive error $v s$ age, separated into breastfed and non-breastfed groups, is presented (Graph 1). From the graph, none of the two groups (breastfed and non-breastfed) was skewed towards any age range, and the two scatter plots were generally well distributed over the ages 6-72 months. It showed that the effect of breastfeeding on refractive 
Distribution of refractive error versus age

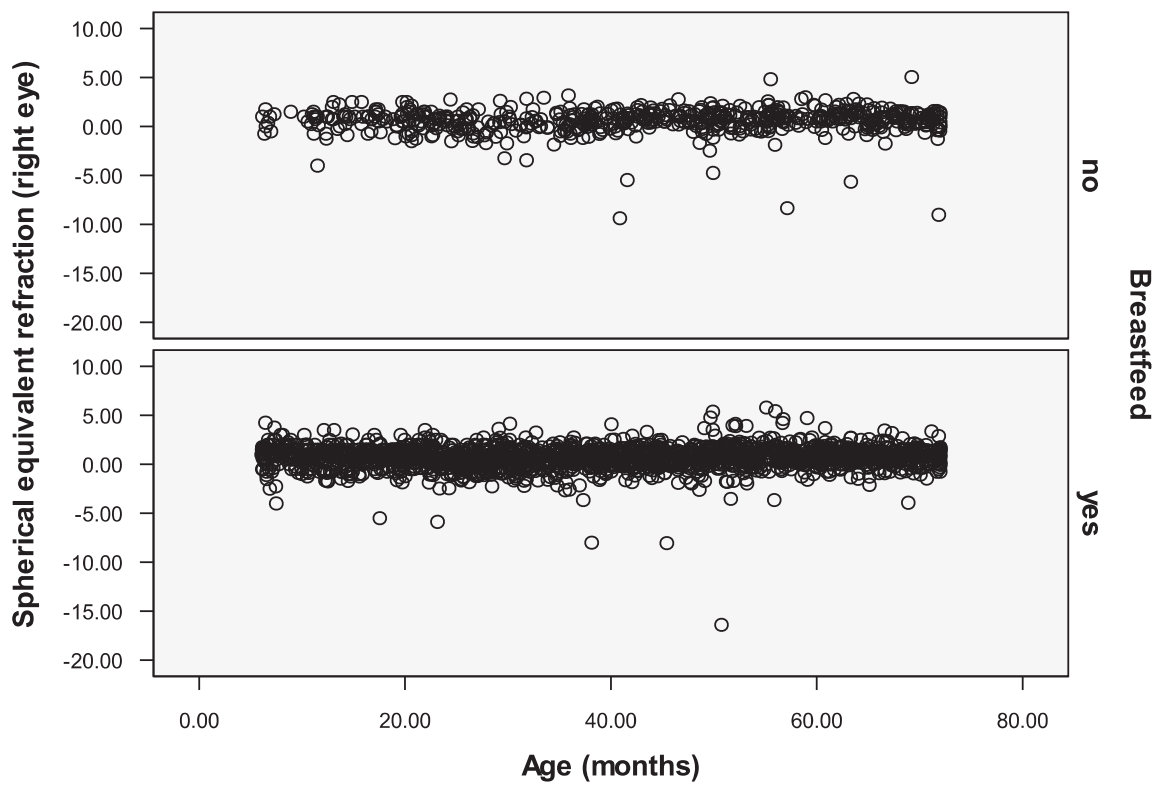

Graph 1 Distribution of refractive error $v$ age.

error was unlikely to be caused by the distribution of our data. Table 1 further illustrated the characteristics of myopes and non-myopes in the STARS study.

\section{Breastfeeding and spherical equivalent refraction}

A history of being breastfed was associated with the spherical equivalent refraction of the right eye. More specifically, children who were breastfed $(0.71 \mathrm{D}, \mathrm{SD} 1.16)$ had a higher mean spherical equivalent refraction; in another words, they were more hyperopic than children who were not breastfed $(0.60 \mathrm{D}, \mathrm{SD} 1.25)(P$-value $=0.05)$. In addition, the duration of breastfeeding (never, $<3$ months, $>3$ months) was associated with spherical equivalent refraction $(P$-value $=0.01)$. When we extended the duration of breastfeeding to 6 months, the results remained significant $(P$-value $=0.04)$. The types and ways of breastfeeding were not associated with the spherical equivalent refraction of the right eye.

For the other characteristics we had tested, only age $(P$-value $=0.01)$ and parental myopia $(P$-value $<0.001)$ were associated with spherical equivalent refraction of the right eye independently. The significance tests for univariate analyses explained that the two variables were statistically related, independent of other risk factors.

The corresponding multivariable regression analysis also showed evidence of association of breastfeeding with spherical equivalent refraction of the right eye. After adjustment with age, gender, outdoor time, mother's education level, mother's smoking history, parental myopia, birth weight, maternal age, and child's height, the mean spherical equivalent refraction of breastfed children was $0.12 \mathrm{D}$ higher than that of children who were not breastfed $(P$-value $=0.03)$ (Table 2$)$. Furthermore, the mean refraction of those children who were breastfed less than 3 months was $0.16 \mathrm{D}$ $(P$-value $=0.01)$ higher than those who were not breastfed and the mean refraction of those who were breastfed more than 3 months were also marginally higher $(0.06 \mathrm{D})$ than those who were not breastfed. However, the latter difference was not statistically significant. The results were similar for those who were breastfed less than 6 months $(0.14 \mathrm{D}, P$-value $=0.02)$ and for those who were breastfed more than 6 months $(0.08 \mathrm{D}, P$-value $=0.26)$, with the non-breastfed children as reference. Children who were exclusively breastfed or directly breastfed had a slightly higher mean refraction ( 0.11 and $0.10 \mathrm{D}$, respectively) than those who were not breastfed, but the difference was not statistically significant with adjustment. For every unit (month) increase in the child's age, the mean refraction will be increased by $0.01 \mathrm{D}$ while controlling other risk factors. This implies an increase in hyperopia with age and it will be a new finding for children aged between 6 and 72 months, which we will circulate in a separate paper. The regression coefficient for the child's height is -0.01 , which implies that for every unit $(\mathrm{cm})$ increase in the child's height, there was a $0.01 \mathrm{D}$ decrease in the mean refraction (a reduction in hyperopia). The analyses for 
Table 1 Characteristics of STARS study by prevalence of myopia (univariate)

\begin{tabular}{|c|c|c|c|c|}
\hline Characteristics & Cases & $\begin{array}{l}\text { With myopia } \\
\quad(\mathrm{n}=299)\end{array}$ & $\begin{array}{l}\text { Without myopia } \\
\qquad(\mathrm{n}=2340)\end{array}$ & P-value \\
\hline Age, mean (SD), months & 2639 & $34.12(17.62)$ & $41.88(18.49)$ & $<0.0001^{\mathrm{a}}$ \\
\hline Gender (\%) & & & & $0.92^{\mathrm{b}}$ \\
\hline Male & 1375 & 11.3 & 88.7 & \\
\hline Female & 1264 & 11.4 & 88.6 & \\
\hline Breastfed (\%) & & & & $0.65^{\mathrm{b}}$ \\
\hline Yes & 2040 & 11.2 & 88.8 & \\
\hline No & 599 & 11.9 & 88.1 & \\
\hline Duration of Breastfeeding (\%) & & & & $0.06^{\mathrm{b}}$ \\
\hline Never & 599 & 11.9 & 88.1 & \\
\hline$<3$ months & 1196 & 9.8 & 90.2 & \\
\hline$>3$ months & 842 & 13.1 & 86.9 & \\
\hline Duration of Breastfeeding (\%) & & & & $0.03^{\mathrm{b}}$ \\
\hline Never & 599 & 11.9 & 88.1 & \\
\hline$<6$ months & 1479 & 10.0 & 90.0 & \\
\hline$>6$ months & 559 & 14.1 & 85.9 & \\
\hline Type of breastfeeding (\%) & & & & $0.80^{\mathrm{b}}$ \\
\hline Never & 599 & 11.9 & 88.1 & \\
\hline Mostly or partly & 1613 & 11.0 & 89.0 & \\
\hline Exclusive & 424 & 11.8 & 88.2 & \\
\hline Ways of breastfeeding (\%) & & & & $0.97^{\mathrm{b}}$ \\
\hline Not breastfed & 599 & 11.9 & 88.1 & \\
\hline Bottled breastfed & 412 & 11.2 & 88.8 & \\
\hline Mixed & 913 & 11.1 & 88.9 & \\
\hline Direct breastfed & 714 & 11.2 & 88.8 & \\
\hline Outdoor time, mean (SD), h/day & 2635 & $0.68(0.89)$ & $0.77(0.98)$ & $0.12^{\mathrm{a}}$ \\
\hline Mother's educational level (\%) & & & & $0.91^{\mathrm{b}}$ \\
\hline None & 11 & 9.1 & 90.9 & \\
\hline Primary & 168 & 11.3 & 88.7 & \\
\hline Secondary & 371 & 11.6 & 88.4 & \\
\hline $\mathrm{O} / \mathrm{N}-\mathrm{level}$ & 550 & 10.5 & 89.5 & \\
\hline A-level & 788 & 10.7 & 89.3 & \\
\hline University & 708 & 12.6 & 87.4 & \\
\hline Others & 14 & 7.1 & 92.9 & \\
\hline Mother's smoking history (\%) & & & & $0.14^{\mathrm{b}}$ \\
\hline Non-smoker & 2364 & 11.7 & 88.3 & \\
\hline Current smoker & 156 & 7.1 & 92.9 & \\
\hline Past smoker & 94 & 8.5 & 91.5 & \\
\hline Parental myopia (\%) & & & & $<0.0001^{\mathrm{b}}$ \\
\hline Non & 757 & 8.9 & 91.1 & \\
\hline Either & 1003 & 9.6 & 90.4 & \\
\hline Both & 874 & 15.6 & 84.4 & \\
\hline Child's height, mean (SD), cm & 2619 & $91.73(13.78)$ & $96.83(13.06)$ & $<0.0001^{\mathrm{a}}$ \\
\hline Birth weight, mean (SD), g & 2568 & $3102(407.4)$ & $3086(459.1)$ & $0.54^{\mathrm{a}}$ \\
\hline Maternal age, mean (SD), years & 2615 & $31.00(4.49)$ & $30.52(4.54)$ & $0.08^{\mathrm{a}}$ \\
\hline $\begin{array}{l}\text { Spherical equivalent of refraction (right eye), } \\
\text { mean (SD), D }\end{array}$ & 2639 & $-1.35(1.54)$ & $0.95(0.78)$ & $<0.0001^{\mathrm{a}}$ \\
\hline Axial length (right eye), mean (SD), mm & 1667 & $22.62(0.93)$ & $22.08(0.71)$ & $<0.0001^{\mathrm{a}}$ \\
\hline
\end{tabular}

Abbreviation: D, dioptre.

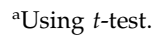

busing $\chi^{2}$-test.

Totals do not add to 2639 cases because of incomplete data for duration of breastfeeding ( 2 missing values), types of breastfeeding ( 3 missing values), ways of breastfeeding (1 missing value), outdoor time (4 missing values), height (20 missing values), birth weight (71 missing values), maternal age (24 missing values), mother's educational level (29 missing values), mother's smoking history ( 25 missing values), parental myopia (5 missing values), axial length (972 missing values). 
Table 2 Factors associated with spherical equivalent refraction (multivariable)

\begin{tabular}{|c|c|c|}
\hline Factors & $\begin{array}{l}\text { Regression coefficient } \\
\left(\beta_{i}\right)(S E)\end{array}$ & P-value \\
\hline Age & $0.01(0.003)$ & $<0.001^{*}$ \\
\hline \multicolumn{3}{|l|}{ Gender } \\
\hline Male & 0 (reference) & \\
\hline Female & $0.06(0.05)$ & 0.18 \\
\hline \multicolumn{3}{|l|}{ Breastfed } \\
\hline No & 0 (reference) & \\
\hline Yes & $0.12(0.06)$ & $0.03^{*}$ \\
\hline Outdoor time & $0.03(0.02)$ & 0.22 \\
\hline \multicolumn{3}{|c|}{ Mother's educational level } \\
\hline None & 0 (reference) & \\
\hline Primary & $-0.003(0.42)$ & 0.99 \\
\hline Secondary & $-0.12(0.41)$ & 0.76 \\
\hline O/N-level & $0.09(0.41)$ & 0.83 \\
\hline A-level & $0.10(0.41)$ & 0.80 \\
\hline University & $0.14(0.41)$ & 0.73 \\
\hline Others & $-0.01(0.51)$ & 0.98 \\
\hline \multicolumn{3}{|c|}{ Mother's smoking history } \\
\hline Non-smoker & 0 (reference) & \\
\hline Current smoker & $0.11(0.10)$ & 0.29 \\
\hline Past smoker & $-0.01(0.12)$ & 0.94 \\
\hline \multicolumn{3}{|l|}{ Parental myopia } \\
\hline Non & 0 (reference) & \\
\hline Either & $-0.15(0.06)$ & $0.01^{*}$ \\
\hline Both & $-0.44(0.06)$ & $<0.001^{*}$ \\
\hline Birth weight & $0.00004(0.00005)$ & 0.41 \\
\hline Maternal age & $-0.003(0.005)$ & 0.53 \\
\hline Child's height & $-0.01(0.005)$ & $0.002^{*}$ \\
\hline
\end{tabular}

The number of cases used in the regression model is 2515 . Those marked with asterisk $\left.{ }^{*}\right)$ has ${ }^{*} P$-value $<0.05$.

height and age of the child with respect to refraction had to be interpreted separately as this study is unique with the age of its participant, being the first study to look at early post-natal children instead of older children in puberty in Singapore.

The prevalence of myopia was lower for children who were breastfed (11.2\%) compared with those who were not $(11.9 \%)$, but the difference was not significant. After adjustment, there seemed to be a lower trend of likelihood of myopia for breastfed children when compared with those who were formula fed (adjusted $\mathrm{OR}=0.85 ; 95 \% \mathrm{CI}(0.62,1.18))$.

\section{Discussion}

In this study, breastfeeding had an association with more positive spherical equivalent refraction of the eye. The breastfeeding status and the duration of breastfeeding were independently associated with the spherical equivalent refraction of the right eye. The mean refractive error for children with a history of being breastfed was significantly more hyperopic than for those who were non-breastfed after adjustment with some socio-demographic factors.

Earlier studies have shown different results for the effect of breastfeeding on myopia. The most relevant publication was a letter by Chong in 2005. In a study of 797 Singapore children aged 10, 11, and 12 years, after adjustment for age, gender, race, mother's educational level, parental myopia, books read per week, household income, birth weight, height, IQ score, and maternal age, the OR for breastfed children was 0.58 with 95\% CI (0.39-0.84). Myopia was defined as the spherical equivalent refraction of at least $-0.5 \mathrm{D}$ and was diagnosed using cycloplegic autorefraction. The mean refraction error of breastfed children $(-1.6 \mathrm{D})$ was higher than the mean of those who were not $(-2.1 \mathrm{D})$ with $P$-value $=0.001 .^{11}$ In contrast to the above study, a recent longitudinal study of British children in 2008 had concluded a statistically insignificant pooled OR for reduced vision $=1.03$ with $95 \% \mathrm{CI}(0.9,1.18)$ and $P$-value for heterogeneity $=0.39$ ( $\chi^{2}$-test) for ages $10-11$ years and pooled OR for reduced vision $=1.02$ with $95 \%$ CI $(0.89$, 1.16) and $P$-value for heterogeneity $=0.88\left(\chi^{2}\right.$-test) for ages $15-16$ years between breastfed and formula-fed children. The child's vision with 6/12 Snellen acuity or worse was considered as poor vision (as an indicator of myopic refractive error). ${ }^{12}$ Possible differences between the Rudnicka et al study and our findings were the difference in ethnicity, definition and diagnosis of refractive errors, proportion of breast milk content for different ethnicity, and the confounders adjusted for in the model, which might be the key elements of differential analyses.

Our findings provided evidence of the relationship between breastfeeding and spherical equivalent refraction, but not for a myopic refraction greater than $-0.5 \mathrm{D}$.

Breast milk consists of polyunsaturated fatty acids (LCPUFA) and anti-oxidants, which are often cited to affect retina development and also influence the growth of the eye and neural development in early life. ${ }^{12}$ This suggested that children who were breastfed might be more resilient to the development of juvenile-onset myopia and thus have a higher mean spherical equivalent refraction. There are several potential reasons for the lack of significant protective effects on a myopia greater than $-0.5 \mathrm{D}$. First, myopia was significantly associated with age, and many studies have shown the same result. ${ }^{1}$ Correspondingly, the children in the STARS study, aged 6-72 months, were still in their initial stage of eye development (emmetropisation). Normally, eye development proceeds from neonatal hypermetropia (long-sighted) towards emmetropia (no refractive error) 
during the development period. Studies have shown that the average refraction at age 3-6 years was approximately $+2 \mathrm{D}$, and the mean refraction for infants was $+2 \mathrm{D} .^{1,2}$ Therefore, the effect of breastfeeding might be concealed by the ocular development at age 6-72 months. Alternatively, the effect of breastfeeding may have an early initial impact on more negative spherical equivalent refraction or the onset of myopia only in older children.

There are some limitations when interpreting our findings. Refractive errors were assessed with three different types of instruments (autokeratorefractometer, streak retinoscopy, or retinomax handheld autorefraction) and the difference in testability of these instruments may affect the accuracy of cycloplegic refraction. Also, the non-participants may be different from the participants in the relationship between breastfeeding and refractive error. This may lead to a distortion of the study results. Confounders such as socioeconomic status and IQ scores may be presented.

The age range of participants between 6 and 72 months is considerably sizeable, especially given that this is the main age range for emmetropisation. I recommend that future population-based studies with larger samples stratify the participants by age, such that the 6- to 12-month-old group is analysed separate from the 1- to 2-year-old group, separate from the 3- to 4-year-old group, and so on. This might help to address the issue of a larger age range, with participants at different stages in the emmetropisation process.

\section{Conclusion}

Children who were breastfed had a higher mean spherical equivalent refraction than children who were fed by formula milk. After adjustment, breastfeeding was associated with more hyperopic spherical equivalent refraction in young Chinese children in Singapore.

\section{Conflict of interest}

The authors declare no conflict of interest.

\section{Acknowledgements}

The STARS project was funded by the National Medical Research Council (NMRC). We also acknowledge the contributions made by the STARS team and the kind participation of all individuals in the STARS project.

\section{References}

1 Curtin MD, Brian J. The Myopias: Basic Science and Clinical Management. Philadelphia: Harper \& Row, 1985.

2 Fredick DR. Myopia. Br Med J 2002; 324: 1195-1199.

3 Morgan I, Rose K. How genetic is school myopia. Prog Retin Eye Res 2005; 24: 1-38.

4 Seet B, Wong TY, Tan DT, Saw SM, Balakrishnan V, Lee LK et al. Myopia in Singapore: taking a public health approach. Br J Ophthalmol 2001; 85: 521-526.

5 Saw S-M, Chua W-H, Hong C-Y, Stone RA, Tan D. Nearwork in early-onset myopia. Invest Ophthalmol Vis Sci 2002; 43: 2 .

6 Heller CD, O'Shea M, Yao Q, Langer J, Langer J, Ehrenkranz RA et al. Human milk intake and retinopathy of prematurity in extremely low birth weight infants. Pediatrics 2007; 120: 19.

7 Hylander MA, Strobino DM, Pezzullo JC, Dhanireddy R. Association of human milk feedings with a reduction in retinopathy of prematurity among very low birthweight infants. J Perinatol 2001; 21: 356-362.

8 Okamoto T, Shirai M, Kokubo M, Takahashi S, Kajino M, Takase $\mathrm{M}$ et al. Human milk reduces the risk of retinal detachment in extremely low birth weight infants. Pediatr Int 2007; 49: 894-899.

9 Williams C, Birch EE, Emmett PM, Northstone K, Avon Longitudinal Study of Pregnancy and Childhood Study Team. Stereoacuity at age $3.5 \mathrm{y}$ in children born full-term is associated with prenatal dietary factors: a report from a population based cohort study. Am J Clin Nutr 2001; 73: 316-322.

10 Singhal A, Morley R, Cole TJ, Kennedy K, Sonksen P, Isaacs $\mathrm{E}$ et al. Infant nutrition and steroacuity at age 4-6y. Am J Clin Nutr 2007; 85: 152-159.

11 Chong Y-S, Liang Y, Tan D, Gazzard G, Stone RA, Saw SM. Association between breastfeeding and likelihood of myopia in children. JAMA 2005; 293(24): 3001-3002.

12 Rudnicka AR, Owen CG, Richards M, Wadsworth ME Strachan DP. Effect of breastfeeding and sociodemographic factors on visual outcome in childhood and adolescence. Am J Clin Nutr 2008; 87: 1392-1399. 